\title{
Die Grenzen der Zielgruppenstrategie
}

\author{
Robin Meyer-Lucht
}

Dieser Beitrag betrachtet die ökonomischen Grenzen der Zielgruppenstrategie in Märkten ausschließlich werbefinanzierter Medienangebote aus Anbietersicht. Es wird modellhaft veranschaulicht, unter welchen Bedingungen ein Anbieter mit Zielgruppenstrategie eine gegenüber dem Reichweitenführer wettbewerbsfähige Position einnimmt. Dabei werden die drei strategischen Eckpfeiler der Zielgruppenstrategie herausgearbeitet: der erforderliche relative Programmaufwand, die durchsetzbaren Aufschläge auf die Tausenderkontaktpreise und die erforderliche relative Reichweite. Diese Einschränkungen der Zielgruppenstrategie werden am Beispiel des Marktgefüges der Online-Nachrichtenangebote diskutiert. Dabei zeigt sich die bobe Bedeutung der Reichweite auch für die Zielgruppenstrategie.

Stichwörter: Zielgruppenstrategie, Tausenderkontaktpreis, Tausenderkontaktkosten, Programmaufwand, Reichweite, Werbefinanzierung, Television Economics

\section{Einleitung: Tausenderkontaktpreise und -kosten}

Die Möglichkeiten und Grenzen der Angebotsvielfalt in Märkten ausschließlich werbefinanzierter Medienangebote sind bislang vornehmlich aus wohlfahrtsökonomischvolkswirtschaftlicher Sicht betrachtet worden. Die Modelle der Television Economics, unter anderem von Steiner (1952), Rothenburg (1962), Owen (1974), Beebe (1977) und Noam (1987), haben hierzu zentrale Ansätze geliefert. Hier erfolgt dagegen nun ein Perspektivwechsel: Die ökonomischen Grenzen für ein breites Angebotsspektrum in ausschließlich werbefinanzierten Medienmärkten werden aus Anbietersicht erörtert.

Ausgangspunkt und Anschauungsmaterial der hier vorgetragenen Überlegungen bilden Online-Nachrichtenangebote. Sie stehen beispielhaft für ausschließlich werbefinanzierte Publikationen ${ }^{1}$ mit sehr niedrigen Verbreitungskosten (vgl. Meyer-Lucht 2005: 53). Die Überlegungen können aufgrund ähnlicher Kostenstruktur leicht auf Hörfunk- und Fernsehangebote übertragen werden. Sie gelten hingegen nicht ohne Weiteres auch für Printmedien mit ihren deutlich höheren variablen Kosten.

Die Geschäftstätigkeit werbefinanzierter Medienangebote besteht, wie hinlänglich erörtert wurde, darin, mit Hilfe kostenfrei zugänglicher Inhalte ein Publikum zu versammeln, dessen Aufmerksamkeit die Medien an Werbetreibende verkaufen (vgl. Owen/ Wildman 1992: 3). Das verkaufsfähige Produkt ausschließlich werbefinanzierter Publikationen ist der „Kontakt“ zu ihren Rezipienten, den sie im Werbemarkt verkaufen.

Diese Rezipientenkontakte werden von den Medienangeboten zu bestimmten Stückkosten hergestellt. Die Kosten eines Kontakts lassen sich einfach ermitteln, indem man die Kosten für die Erstellung der Inhalte, den Programmaufwand ${ }^{2}$, durch die

1 Kommerzielle Online-Nachrichtenangebote, wie Spiegel Online oder FAZ.net, finanzieren sich im strengen Sinne nicht ausschließlich durch Werbung, sondern erzielen einen geringen Umsatzanteil aus anderen Quellen (vgl. Meyer-Lucht 2005: 48). Für die modellhafte Betrachtung ist es jedoch zulässig, Online-Nachrichtenangebote als werbefinanziert zu betrachten.

2 Die Formulierung Programmaufwand wird hier verwendet, um medienübergreifend den Aufwand von Publikationen für Inhalte zu bezeichnen. 
Anzahl der Rezipienten (die Reichweite) teilt. Zur besseren Vergleichbarkeit mit der Verkaufspraxis im Werbemarkt ist es sinnvoll, die Kosten für die Herstellung von tausend Kontakten, die „Tausenderkontaktkosten“ (TKK) (vgl. Heinrich 1999: 315), zu bestimmen:

TKK $=\frac{\text { Programmaufwand }}{(1.000) \text { Rezipienten }}$

Den Tausenderkontaktkosten steht der Preis gegenüber, den tausend Kontakte im Werbemarkt erzielen: der „Tausenderkontaktpreis“ (TKP) (vgl. Wirtz 1994: 143). Fragen der Werbeplatz-Auslastung werden hier ausgeklammert. Es wird modellhaft davon ausgegangen, dass jeder Rezipient einen Kontakt erzeugt und dieser Kontakt auch im Werbemarkt abgesetzt wird. ${ }^{3}$

Das Ziel aller werbefinanzierten Medien ist es, eine Marktposition, eine Reichweite, ein Publikum und einen Programmaufwand zu finden, bei dem die erzielten Werbeschaltpreise (TKPs) komfortabel über den Stückkosten ihrer Herstellung (TKKs) liegen. Sie maximieren ihren Gewinn, indem sie den Abstand von TKKs und TKPs und/oder ihre Reichweite maximieren.

Um ihre TKKs zu minimieren, setzen die Medien auf die für Medienmärkte typische Fixkostendegression. Sie versuchen, die Nachfrage möglichst umfassend zu bündeln, um auf diese Weise die hohen Fixkosten für die Herstellung der Inhalte auf möglichst viele Kontakte zu verteilen. Dabei werden sie ihren Programmaufwand so lange ausweiten, wie die damit verbundenen Reichweitensteigerungen die TKKs senken. Zugleich werden sie stets überprüfen, wo sie ihren Programmaufwand reduzieren können, ohne Rezipienten zu verlieren. Nicht eine möglichst hohe Reichweite ist damit das Ziel der Publikationen, sondern eine Kombination aus Reichweite und Programmaufwand, die jeweils die niedrigsten TKKs in einem (hinreichend großen) Marktsegment erlaubt (vgl. Litman 1988: 29; Heinrich 1999: 285).

Die Fixkostendegression nivelliert dabei die Nachfrage der Rezipienten erheblich (vgl. Heinrich 1999: 122): Die Kostenvorteile der Massenproduktion wirken sich bei ausschließlich werbefinanzierten Publikationen nicht direkt über niedrigere Verkaufspreise im Rezipientenmarkt aus, sondern indirekt über die Höhe des finanzierbaren Programmaufwands. Reichweitenstarke Publikationen können auf Basis ihrer Kostenvorteile einen erheblich höheren Programmaufwand treiben als spezifische Kleinserien-Publikationen.

Die Rezipienten werden aber nur einen bestimmten Abstand im Programmaufwand zwischen einer Publikation, die ihren Präferenzen eher entspricht, und einer Massenpublikation akzeptieren ${ }^{4}$. Häufig werden sie sich dem Massenprodukt zuwenden, weil sie den höheren Programmaufwand dem Differenzierungsvorteil der kleinen Publikation vorziehen. Auf diese Weise „nivelliert“ die hohe Fixkostendegression die ursprünglich heterogene Nachfrage in Rezipienten- und Werbemarkt erheblich (vgl. Owen/Wildman 1992: 339) und bündelt sie in wenigen Publikationen. Dabei verhindert die Heteroge-

3 Weitere Werbe-Vergütungsformen, wie transaktionsbasierte Modelle, werden ebenfalls nicht berücksichtigt.

4 Auch wenn der Qualitätswettbewerb unter Medienprodukten durch Informationsasymmetrien eingeschränkt ist (vgl. Heinrich 1996), so ist doch glaubhaft, dass der Rezipient erhebliche Unterschiede im Programmaufwand zweier ähnlicher Publikationen wahrzunehmen vermag und dies seine Wahl beeinflusst. 
nität der Nachfrage jedoch zumeist die Dominanz eines einzelnen Anbieters. Vielmehr bilden sich differenzierte Produktmärkte heraus, bei denen eine Anzahl von Firmen dieselbe „Art“ von Informationen in verschiedenen Varianten herstellt (vgl. Shapiro/Varian 1999: 42).

\section{Reichweiten- und Zielgruppenstrategie}

Ausgangspunkt für die Strategiefindung werbefinanzierter Medienangebote ist die Nachfrage im Werbemarkt, denn allein hier erzielen sie Umsätze. Im Werbemarkt konkurrieren die Angebote auf der Ebene der „Kontaktqualität“. Diese bemisst sich vornehmlich nach folgenden Eigenschaften (vgl. Detering 2001: 62; Heinrich 1996: 79; Unger 2002: 7f.; Koschat/Putsis 2000): der Reichweite des Werbeträgers, der qualitativen Zusammensetzung seiner Rezipienten, dem Image der die Werbebotschaft umgebenden Medieninhalte, der technischen Eignung des Mediums und den Kosten der Platzierung.

Auch im Werbemarkt finden die generischen Wettbewerbsstrategien der Kostenführerschaft und der Differenzierung ihre Anwendung (vgl. Porter 1990: 67; Müller-Stewens/Lechner 2001: 105; zu Kombinationsmöglichkeiten: Kuss/Tomczak 2002: 86f.). $\mathrm{Zu}$ beachten ist dabei stets die enge Verschränkung der Angebotspolitik in Werbe- und Rezipientenmarkt.

Die Strategie der Kostenfübrerschaft im Werbemarkt müsste eigentlich darauf zielen, auf Basis der niedrigsten Tausenderkontaktkosten (TKKs) die günstigsten Werbepreise (TKPs) anbieten zu können (vgl. Heinrich 1999: 315). Der Schlüssel zu einer sinnvollen Senkung der Stückkosten liegt in Medienmärkten mit niedrigen Verbreitungskosten, wie bereits erörtert, in einer Ausweitung der Reichweite (vgl. Shapiro/Varian 1999: 47). Der Strategieansatz der Kostenführerschaft mutiert daher in Medienmärkten mit niedrigen Verbreitungskosten zu einer Strategie der Reichweitenfübrerschaft. Eine hohe Reichweite gewährt dabei nicht nur hohe Stückkostenvorteile, sondern macht den Werbeträger zugleich besonders im Sinne der oben beschriebenen Nachfrage im Werbemarkt. Durch diese Kombination von Kosten- und Differenzierungsvorteil bietet die Strategie der Reichweitenführerschaft einen doppelten Schutz vor den Kräften des Branchenwettbewerbs (vgl. Porter 1990: 66). Sie kann daher als robuste Strategie in Medienmärken gelten, ist jedoch mit einem hohen Anfangs-Investitionsrisiko verbunden.

Die Grenze der Reichweitenstrategie bildet die Heterogenität der Nachfrage in Werbe- und Rezipientenmarkt, da sich diese zumeist nicht von einer Publikation allein bündeln lässt. Günstig für diesen Strategieansatz ist daher eine weitgehend homogene, der Normalverteilung ähnliche Verteilung der Präferenzen insbesondere im Rezipientenmarkt (vgl. Beebe 1977; Schatz 1994).

Die klassische Differenzierungsstrategie in Märkten ausschließlich werbefinanzierter Medienangebote ist die Zielgruppenstrategie. Hierbei hebt sich eine Publikation gegenüber dem Reichweitenführer durch eine im Sinne der Werbetreibenden besondere Zusammensetzung des Publikums hervor. Vom Werbemarkt werden insbesondere wohlhabende, hoch gebildete und junge Zielgruppen nachgefragt, da ihre Konsumneigung als höher gilt. Für den Zugang zu diesen Rezipientengruppen sind die Werbetreibenden bereit, deutlich höhere TKPs zu zahlen (vgl. Koschat/Putsis 2000). Die Zielgruppenstrategie setzt auf die Durchsetzung eines höheren TKPs auf Basis eines besonderen Publikums.

Die Zielgruppenstrategie bündelt eine Nachfrage, die vom Massenangebot nicht bedient werden kann. Sie setzt damit auf eine Heterogenität der Nachfrage in Werbe- und Rezipientenmarkt. Dabei steigert die Minderheitenbevorzugung durch die Werbekun- 
den die Attraktivität bestimmter Rezipientensegmente. Die Heterogenität der Rezipientennachfrage wird somit durch die Nachfrage im Werbemarkt selektiv verstärkt (vgl. Beebe 1977). Der für bestimmte Rezipientengruppen getriebene Aufwand steigt. Die Zielgruppenstrategie erlaubt daher ein durch die Interessen der Werbekunden vermitteltes zusätzliches Programmangebot.

Die Zielgruppenstrategie stellt den zentralen Mechanismus dar, in Märkten ausschließlich werbefinanzierter Medien Minderheitenangebote zu machen. Ihre Grenzen sind zugleich auch die Grenzen der Heterogenität des Programmangebots in diesen Märkten.

\section{Die drei strategischen Eckpfeiler der Zielgruppenstrategie}

Bei der Zielgruppenstrategie greifen Differenzierungsstrategien in Werbe- und Rezipientenmarkt ineinander: Das besondere Publikum wird durch besondere Inhalte versammelt. Sie folgt damit den erprobten Mechanismen der Produktdifferenzierung. Sie bietet damit guten Schutz vor den Kräften des Branchenwettbewerbs. Sie ist allerdings nicht so robust wie die Reichweitenstrategie. Ein Anbieter mit Zielgruppenstrategie muss seine Marktposition stets dahingehend überprüfen, ob er eine im Vergleich zum Reichweitenführer wettbewerbsfähige Position einnimmt.

Aufgrund der hohen Fixkostendegression in Medienmärkten geraten Anbieter mit Zielgruppenstrategie gegenüber dem Reichweitenführer rasch uneinholbar in Stückkostennachteile. Ihr Stückkostennachteil übersteigt dann ihren Differenzierungsvorteil. Konkret drohen die Tausenderkontaktkosten (TKKs) das Niveau der durchsetzbaren Tausenderkontaktpreise (TKPs) zu übersteigen. Die Anbieter mit Zielgruppenstrategie können auf diese Situation reagieren, indem sie auf Basis ihres differenzierenden Publikums möglichst hohe Kontaktpreise im Werbemarkt durchsetzen oder ihren Programmaufwand reduzieren.

Anbieter mit Zielgruppenstrategie müssen ferner darauf achten, eine gewisse Reichweitenschwelle nicht zu unterschreiten. Andernfalls können sie im Werbemarkt kein relevantes Kontaktvolumen anbieten und werden von den Werbetreibenden nur eingeschränkt wahrgenommen.

Der Zielgruppenstrategie sind damit drei Grenzen gesetzt:

1. Die Höhe der Aufschläge auf die Tausenderkontaktpreise, die ein Anbieter mit Zielgruppenstrategie im Vergleich zum Reichweitenführer durchzusetzen vermag. Koschat und Putsis haben errechnet, dass Werbetreibende für besonders wohlhabende und konsumaffine Publikumssegmente den bis $\mathrm{zu}$ siebenfachen Preis bezahlen (vgl. Koschat/Putsis 2000). Für ihr gesamtes Publikum können Zielgruppenanbieter in der Praxis zumeist keine Aufschläge in der genannten Höhe gegenüber dem Reichweitenführer durchsetzen, wie sich unten zeigen wird.

2. Die Höbe des relativen Programmaufwands, der erforderlich ist, um den Differenzierungsansatz des Zielgruppenangebotes im Rezipientenmarkt gegenüber dem Angebot des Reichweitenführers zu stützen. Ist der Programmaufwand der Zielgruppenpublikation zu niedrig bemessen, werden sich die Rezipienten trotz anders gelagerter Vorlieben dem aufwändigeren Angebot des Reichweitenführers zuwenden.

3. Die Höhe der relativen Reichweite, die erforderlich ist, um unter Ausnutzung der Fixkostendegression die Tausenderkontaktkosten des erforderlichen Programmaufwands unterhalb des Niveaus der durchsetzbaren Tausenderkontaktpreise zu senken und zugleich ein relevantes Kontaktvolumen im Werbemarkt anbieten zu können.

Hiermit sind die drei zentralen strategischen Eckpfeiler der Zielgruppenstrategie be- 
nannt: die Höhe der durchsetzbaren Aufschläge auf die Werbepreise, die Höhe des erforderlichen relativen Programmaufwands und die Höhe der benötigten relativen Reichweite.

\section{Modellierung der Grenzen der Zielgruppenstrategie}

Die Grenzen der Zielgruppenstrategie durch die drei benannten strategischen Eckpfeiler lassen sich modellhaft skizzieren. Hierfür wird vereinfacht angenommen, dass alle Anbieter über die gleiche Auslastung der Werbeplätze verfügen, die gleiche Umsatzrendite anstreben und Verbreitungskosten vernachlässigt werden können. Verglichen werden der Programmaufwand PA, der Tausenderkontaktpreis WP und die Reichweite RW eines Reichweitenführers R und eines Anbieters mit Zielgruppenstrategie Z. Für beide gilt folgende Kalkulation:

Programmanfwand $P A+$ Gewinnaufschlag $=$ Tausenderkontaktpreis WP * Reichweite $R W$

Unter der Voraussetzung, dass beide Anbieter die gleiche Umsatzrendite anstreben, muss das Verhältnis von Umsatzerlösen (WP * RW) und Kosten PA bei beiden gleich sein, weshalb gilt:

$\frac{\mathrm{PAz}}{\mathrm{PAR}}=\frac{\mathrm{WPz}}{\mathrm{WPR}} * \frac{\mathrm{RWz}}{\mathrm{RWR}}$

Das Verhältnis $\frac{P A z}{P A_{R}}$ bezeichnet den Programmaufwand, den ein Anbieter mit Zielgruppenstrategie im Verhältnis zum Reichweitenführer zu finanzieren vermag. Das Verhältnis $\frac{W_{2}}{W P_{R}}$ bezeichnet die Höhe des vom Zielgruppenanbieter durchsetzbaren Aufschlags auf die Tausenderkontaktpreise und das Verhältnis $\frac{\mathrm{RW} z}{\mathrm{RW}_{\mathrm{R}}}$ seine relative Reichweite. Die Formel zeigt die Grenzen der Zielgruppenstrategie auf, wenn die gleiche Umsatzrendite wie beim Reichweitenführer erzielt werden soll:

Finanzierbarer relativer Programmanfwand der Zielgruppenpublikation = durchsetzbarer TKP-Aufschlag * relative Reichweite

Diese Einschränkungen der Zielgruppenstrategie lassen sich mit Hilfe einer Skizze veranschaulichen (vgl. Abbildung 1). Hierzu wird die Reichweite an der Abszisse und der durchsetzbare Tausenderkontaktpreis an der Ordinate angetragen. In das Koordinatensystem wird die Position des Reichweitenführers R mit einer Reichweite von 100 und einem TKP von 1 eingetragen. Auf dieser Basis lässt sich der Positionierungsrahmen für Anbieter mit Zielgruppenstrategie erörtern. Linie A skizziert die möglichen Kombinationen von Werbekontaktpreis und Reichweite, die ein Anbieter mit Zielgruppenstrategie erreichen muss, wenn er den gleichen Programmaufwand wie der Reichweitenführer finanzieren will.

Linie B kennzeichnet mögliche Kombinationen von Kontaktpreis und Reichweite, die ein Anbieter mit Zielgruppenstrategie erreichen muss, will er den halben Programmaufwand des Reichweitenführers finanzieren. Es wird deutlich, dass im Bereich niedriger Reichweiten das Umfeld für Anbieter mit Zielgruppenstrategie sehr schwierig wird: Sie müssen entweder sehr hohe Kontaktpreise durchsetzen oder mit einem sehr geringen Programmaufwand auskommen. 
Abbildung 1: Positionierungsrabmen für Anbieter mit Zielgruppenstrategie

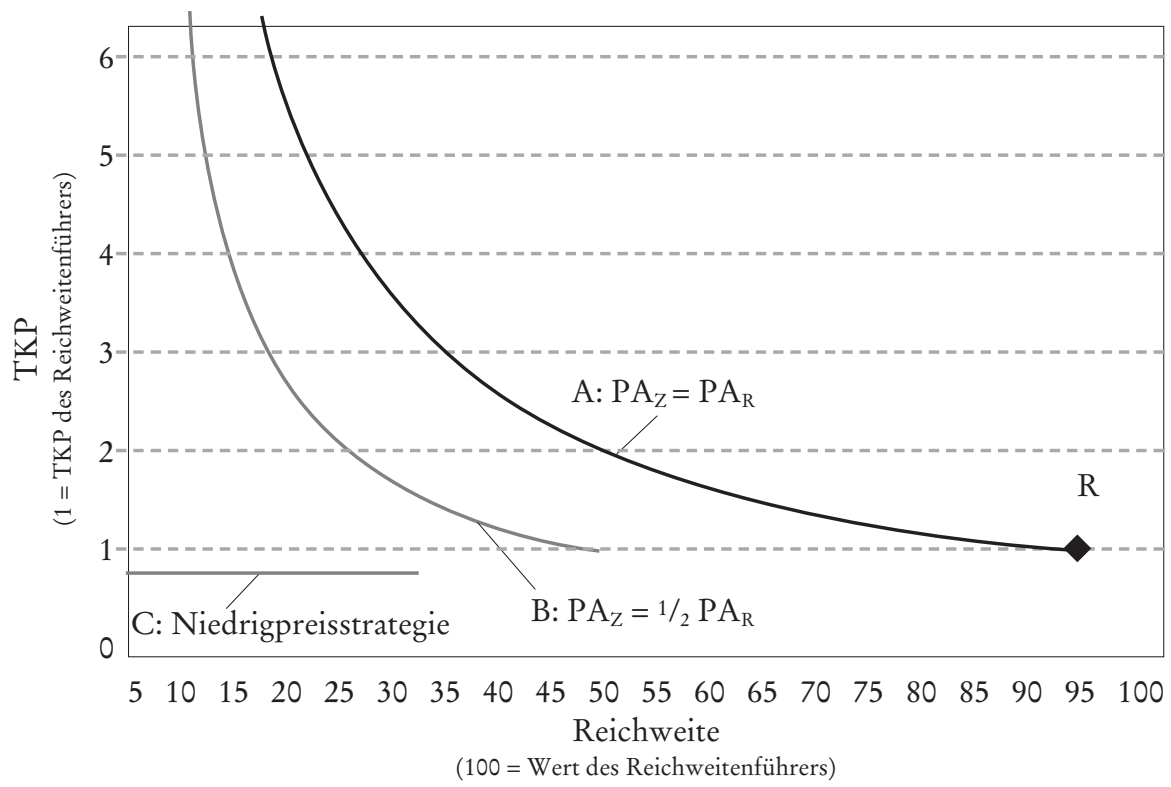

Linie C zeigt die Niedrigpreis-Politik eines Anbieters mit der Strategie einer Produktionskostenfübrerschaft. Ein solcher Ansatz wird von Anbietern gewählt, die schlecht für den Werbemarkt positioniert und daher gezwungen sind, sich vornehmlich über den Preis hervorzuheben. Um die Stückkosten trotz niedriger Reichweite gering zu halten, muss ein solcher Anbieter mit einem sehr geringen Programmaufwand auskommen. ${ }^{5}$ Er muss daher fürchten, dass seine Rezipienten zu einem aufwändigeren Programmangebot abwandern. Er ist schlecht für Preiskämpfe im Werbemarkt mit reichweitenstärkeren Anbietern gerüstet. Diese Strategie schützt daher verhältnismäßig schlecht vor den Kräften des Branchenwettbewerbs.

\section{Anwendung auf die gegenwärtigen Online-Nachrichtenangebote}

Der in Abbildung 1 dargestellte Positionierungsrahmen lässt sich nun auf konkrete Situationen anwenden. Beispielhaft wird hier die Konstellation im Markt der OnlineNachrichtenangebote betrachtet. Für den Vergleich wurden exemplarisch fünf Publikationen mit unterschiedlichen Marktpositionen ausgewählt. Sie können jeweils als profilierte Vertreter ihrer strategischen Gruppe gelten.

Abbildung 2 zeigt die Reichweiten und die Kontaktpreise der Online-Nachrichtenangebote Bild.T-Online, FAZ.net, Netzeitung, Spiegel Online und T-Online Nachrich-

5 Anbieter mit Produktionskostenführerschaft werden mit ihrer Niedrigpreisstrategie häufig auch auf eine hohe Auslastung ihrer Werbeplätze setzen. Diesem Ansatz sind jedoch durch begrenzten Platz und die Akzeptanz der Rezipienten enge Grenzen gesetzt. Die Auslastung der Werbeplätze wurde aus Gründen der Einfachheit aus dem hier erörterten Modell ausgeklammert. 


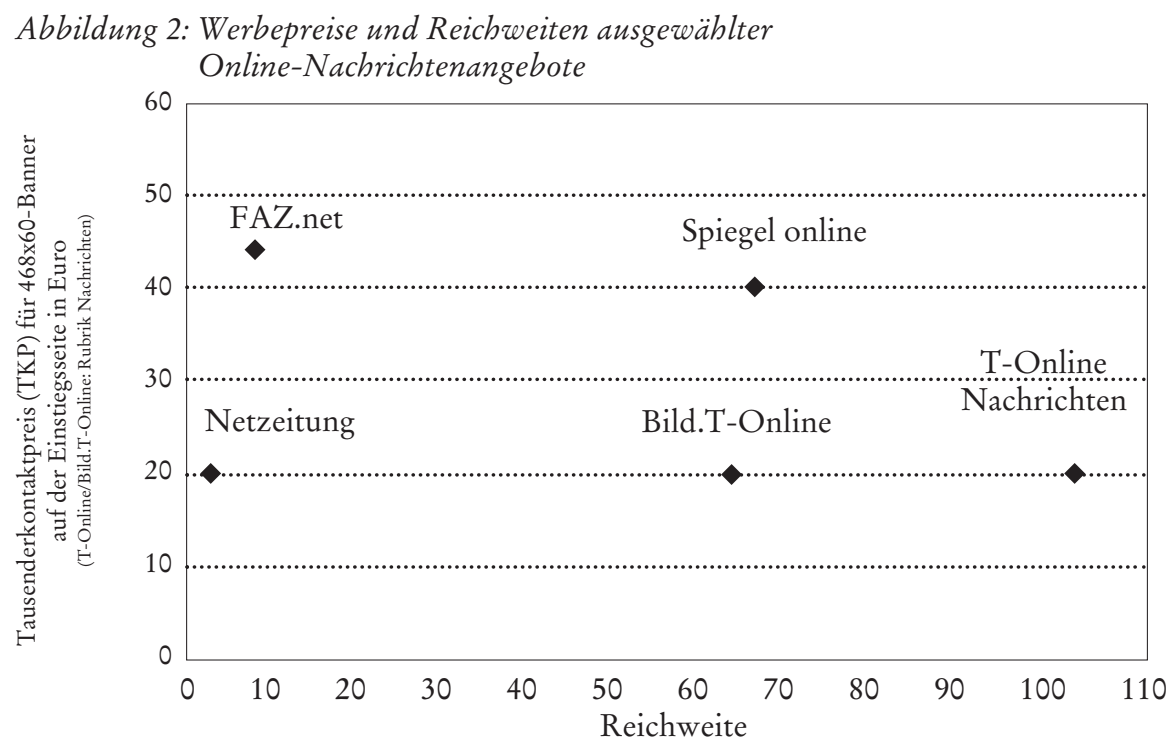

Quellen: IVW April 2004 / Netzeitung / Nielsen Netratings Mai 2004/ ACTA 20046, Preislisten der Anbieter; Stand April 2004

ten. Die Werbepreise wurden den offiziellen Preislisten der Publikationen entnommen. Zwar werden die Anbieter in den allermeisten Fällen ihren Werbekunden erhebliche Rabatte einräumen. Die Listenpreise können jedoch als Signale verstanden werden, die Rückschlüsse auf ihre Preisstrategien zulassen.

Abbildung 2 zeigt nahezu idealtypisch die zuvor theoretisch in Abbildung 1 erörterte Situation: Das Portalangebot T-Online Nachrichten ist Reichweitenführer unter den Online-Nachrichtenangeboten. Aus dieser Position heraus verlangt die Site einen TKP von 20 Euro.

Demgegenüber setzt Spiegel Online auf eine Zielgruppenstrategie in einem breiter gefassten Qualitätssegment des Publikums. Das Angebot vermag die von der Werbeindustrie nachgefragten Zielgruppen in höherem Maße zu versammeln als T-Online Nachrichten und insbesondere Bild.T-Online (vgl. Tabelle 1), verfügt über eine erhebliche Reichweite und ein imageträchtiges redaktionelles Umfeld. Auf dieser Basis verlangt Spiegel Online einen doppelt so hohen TKP wie der Reichweitenführer.

Die hohe Reichweite stützt die Zielgruppenstrategie komfortabel: Würden Spiegel Online und T-Online Nachrichten über die gleiche Auslastung verfügen und die ge-

6 Für Spiegel Online, FAZ.net und Bild.T-Online lagen Daten der Informationsgesellschaft zur Feststellung der Verbreitung von Werbeträgern (IVW) vor. Ihre Reichweitendaten wurden auf Basis gleicher Gewichtung von Visits und Page Impressions ermittelt. Von der Netzeitung lagen Eigenangaben zu den Page Impressions vor, die nach dem Durchschnitt der anderen Sites um Visits ergänzt wurden. Für den Portalbereich T-Online Nachrichten lagen keine gesonderten IVW-Daten vor. Auf Basis der Ergebnisse von Nielsen Netratings und Allensbacher Computer- und Technikanalyse (ACTA 2004) wurde ein Reichweitenvorsprung von 50 Prozent gegenüber Spiegel Online taxiert (vgl. Meyer-Lucht 2005: 40). Für den konzeptionellen Vergleich ist die Datenqualität damit ausreichend. 
Tabelle 1: Die Zusammensetzung des Publikums der Online-Nachrichtenangebote aus Abbildung 2

\begin{tabular}{lccc}
\hline & $\begin{array}{l}\text { Abgeschlossenes } \\
\text { Studium }\end{array}$ & $\begin{array}{l}\text { Haushaltsnettoeinkommen } \\
\text { von 4.000 Euro und mehr }\end{array}$ & $\begin{array}{l}\text { Leitende Angestellte/ } \\
\text { Beamte/Inhaber/ } \\
\text { freie Berufe }\end{array}$ \\
\hline Bevölkerungsschnitt & $15 \%$ & $11 \%$ & $13 \%$ \\
Bild.T-Online & $18 \%$ & $13 \%$ & $20 \%$ \\
T-Online Nachrichten & $25 \%$ & $19 \%$ & $26 \%$ \\
Spiegel Online & $36 \%$ & $22 \%$ & $32 \%$ \\
FAZ.net & $43 \%$ & $29 \%$ & $32 \%$ \\
\hline
\end{tabular}

Quelle: Allensbacher Computer- und Technikanalyse 2004 (ACTA); angegeben ist der Prozentsatz der Nutzer pro Woche (NpW) zwischen 30 und 59 Jahren, auf die das jeweilige Merkmal zutrifft; die Netzeitung nimmt nicht an der ACTA teil

nannten Preise tatsächlich erzielen, so könnte Spiegel Online bei gleicher Umsatzrendite einen höheren Programmaufwand finanzieren als der Reichweitenführer.

Bei FAZ.net zeigen sich dagegen die Schwierigkeiten einer sehr eng gefassten Zielgruppenstrategie. Zwar kann die Site als „Netz der klugen Köpfe“ ein im Sinne der Werbetreibenden noch differenzierteres Publikum versammeln als Spiegel Online (vgl. Tabelle 1). Doch wertet dies die Site offenbar nicht stark genug auf, um einen deutlich höheren TKP durchzusetzen. Hier zeigt sich, dass die Höhe des durchsetzbaren TKPAufschlags eng begrenzt ist.

Mit einem nur geringfügig höheren TKP als Spiegel Online kann FAZ.net jedoch den eigenen reichweitenbedingten Kostennachteil nicht ausgleichen und muss mit einem erheblich geringeren Programmaufwand auskommen: Würden beide Sites über die gleiche Auslastung der Werbeplätze verfügen und würden sie die verlangten Preise tatsächlich erzielen, so könnte Spiegel Online den siebenfachen Programmaufwand finanzieren. Bei derart großen Unterschieden werden sich viele Rezipienten dem ressourcenstärkeren Angebot Spiegel Online zuwenden, auch wenn FAZ.net ihren Präferenzen eher entspricht. Hier zeigt sich, wie die Fixkostendegression die ursprünglich heterogenere Nachfrage nivelliert.

Abbildung 2 zeigt auch die Niedrigpreis-Strategie der Netzeitung. Sie verortet sich vom Publikum her unter den Zielgruppenpublikationen, verlangt aber dennoch einen Tausenderkontaktpreis auf dem Niveau der Massenanbieter T-Online Nachrichten und Bild.T-Online. Sie unterbietet Spiegel Online und FAZ.net damit deutlich. Die Site tritt im Werbemarkt preisaggressiv auf und setzt darauf, über eine hohe Werbeplatz-Auslastung ihre Umsatzerlöse zu maximieren. Ihre geringe Reichweite bedeutet dabei jedoch in jedem Fall, dass sie mit einem geringen Programmaufwand auskommen muss. Sie ist gezwungen, die Strategie der Produktionskostenführerschaft zu wählen, was sie nur eingeschränkt vor den Kräften des Branchenwettbewerbs schützt.

\section{Fazit}

Der vorliegende Beitrag hat die Grenzen der Zielgruppenstrategie in Märkten ausschließlich werbefinanzierter Publikationen aus Sicht der Anbieter zunächst modellhaft und dann am konkreten Beispiel erörtert. Deutlich wurden die drei strategischen Eckpfeiler der Zielgruppenstrategie: die Höhe der durchsetzbaren Aufschläge auf die Tau- 
senderkontaktpreise, die Höhe des relativen Programmaufwands und die Höhe der erforderlichen relativen Reichweite.

Zwar können Anbieter mit Zielgruppenstrategie ihre Stückkostennachteile teilweise durch Aufschläge auf die Tausenderkontaktpreise ausgleichen. Da die Höhe dieser Aufschläge jedoch eng begrenzt ist, müssen Anbieter ihre Zielgruppenstrategie mit einer erheblichen Reichweite kombinieren - oder mit einem sehr geringen Programmaufwand auskommen. In letzterem Fall droht ihnen, ihre Rezipienten an ressourcenstärkere $\mathrm{Pu}-$ blikationen zu verlieren.

Das Beispiel der Online-Nachrichtenangebote konnte diese Grenzen verdeutlichen. Nur in einer ausgewogenen Kombination aus Differenzierungsansatz im Rezipientenund Werbemarkt sowie einer hohen Reichweite im jeweiligen Nachfragesegment erweist sich die Zielgruppenstrategie als eine robuste Strategie, die vor den Kräften des Branchenwettbewerbs zu schützen vermag.

\section{Literatur}

Beebe, Jack H. (1977): Institutional Structure and Program Choices in Television Markets. Quarterly Journal of Economics 91 (1), S. 15-37.

Detering, Dietmar (2001): Ökonomie der Medieninhalte: Allokative Effizienz und soziale Chancengleichheit in den Neuen Medien. Münster: Lit.

Heinrich, Jürgen (1996): Qualitätswettbewerb und/oder Kostenwettbewerb im Mediensektor. Rundfunk \& Fernsehen 44 (2), S. 165-184.

Heinrich, Jürgen (1999): Medienökonomie. Band 2: Hörfunk und Fernsehen. Opladen: Westdt. Verl.

Koschat, Martin A. und Putsis, William P. (2000): Who Wants You When You're Old and Poor? Exploring the Economics of Media Pricing. Journal of Media Economics 13 (4), S. 215-232.

Kuss, Alfred und Tomczak, Torsten (2002): Marketingplanung: Einführung in die marktorientierte Unternehmens- und Geschäftsfeldplanung. 3. Aufl. Wiesbaden: Gabler.

Litman, Barry R. (1988): Microeconomic Foundations. In: Picard, Robert G. et al. (Hg.): Press Concentration and Monopoly: New Perspectives on Newspaper Ownership and Operation. Norwood, NJ: Ablex.

Meyer-Lucht, Robin (2005): Nachrichtensites im Wettbewerb. München: R. Fischer.

Müller-Stewens, Günter und Lechner, Christoph (2001): Strategisches Management: Wie strategische Initiativen zum Wandel führen. Stuttgart: Schäffer-Poeschel.

Noam, Eli M. (1987): A Public and Private-Choice Model of Broadcasting. Public Choice 55, S. $163-187$.

Owen, Bruce M. et al. (1974): Television Economics. Lexington, MA: Heath.

Owen, Bruce M. und Wildman, Steven S. (1992): Video economics. Cambridge, Mass. [u. a.]: Harvard Univ. Press.

Porter, Michael E. (1990): Wettbewerbsstrategie. 6. Aufl. Frankfurt am Main: Campus.

Rothenberg, Jerome (1962): Consumer Sovereignty and the Economics of TV Programming. Studies in Public Communication 4, S. 45-54.

Schatz, Heribert (1994): Das duale Rundfunksystem in modernisierungstheoretischer Sicht, in: Holgersson, Silke et al. (Hg.): Dualer Rundfunk in Deutschland: Beiträge zur Theorie der Rundfunkentwicklung. Münster: Lit.

Shapiro, Carl und Varian, Hal R. (1999): Online zum Erfolg. München: Langen Müller/Herbig.

Steiner, Peter O. (1952): Program Patterns and Preferences, and the Workability of Competition in Radio Broadcasting. Quarterly Journal of Economics 66, S. 194-233.

Unger, Fritz et al. (Hg.) (2002): Mediaplanung. Heidelberg: Physica-Verl.

Wirtz, Bernd W. (1994): Neue Medien, Unternehmensstrategien und Wettbewerb im Medienmarkt: eine wettbewerbstheoretische und -politische Analyse. Frankfurt am Main: Europ. Verl. der Wissenschaft. 